\title{
氧化偶联反应和酸催化反应制备活性买麻藤醇二聚体衍生物
}

\author{
姚春所林 茂* 杨庆云 \\ (中国医学科学院 北京协和医学院 药物研究所 天然药物活性物质与功能国家重点实验室 北京 100050)
}

\begin{abstract}
摘要 以天然得到的买麻藤醇为原料, 以 $\mathrm{FeCl}_{3} \cdot 6 \mathrm{H}_{2} \mathrm{O}$ 为氧化剂进行氧化偶联反应和酸催化二聚反应, 获得了 2 个新的 买麻藤醇二聚体及一个新的苯基菜衍生物: 4-[1-(2,6-二羟基苯基)-2-(3,5-二羟基苯基)乙基]-2-[(1E)-2-(3,5-二羟基苯基) 乙烯基]-1,3-苯二醇(1)，2-[1-(2,6-二羟基苯基)-2-(3,5-二羟基苯基)乙基]-5-[(1E)-2-(2,6-二羟基苯基)乙烯基]-1,3-苯二醇 (2)和 4-(6,8-二甲氧基-2-菜基)-1,3-苯二醇(3). 应用波谱分析的方法确定了它们的结构, 并分别讨论了它们可能的形成 机理. 其中, 化合物 $\mathbf{1}$ 和 $\mathbf{2}$ 首次为人工合成的二苯乙烯链状二聚体. 活性测试结果表明, 化合物 $\mathbf{1 , 2}$ 和 3 显示有较强的 抗氧化活性, 其 $\mathrm{IC}_{50}$ 值分别为 $6.29 \times 10^{-9}, 4.19 \times 10^{-6}$ 和 $2.96 \times 10^{-5} \mathrm{~mol} \cdot \mathrm{L}^{-1}$; 化合物 2 还显示有较强的抗炎活性. 关键词 买麻藤醇二聚体; 氧化偶联反应; 生物活性
\end{abstract}

\section{Preparation of Active Gnetol Dimers by Oxidative Coupling Reaction and Acid-Catalyzed Dimerization}

\author{
Yao, Chunsuo Lin, Mao* Yang, Qingyun \\ (State Key Laboratory of Bioactive Substance and Function of Natural Medicines, Institute of Materia Medica, \\ Chinese Academy of Medical Sciences and Peking Union Medical College, Beijing 100050)
}

\begin{abstract}
Oxidative coupling reaction with $\mathrm{FeCl}_{3} \cdot 6 \mathrm{H}_{2} \mathrm{O}$ as oxidant and acid-catalyzed dimerization of natural gnetol in methanol afforded two new gnetol dimers and one new phenyl naphthalene derivative: 4-[1-(2,6-dihydroxyphenyl)-2-(3,5dihydroxyphenyl)ethyl]-2-[(1E)-2-(3,5-dihydroxyphenyl)ethenyl]-1,3-benzenediol (1), 2-[1-(2,6-dihydroxyphenyl)-2-(3,5-dihydroxyphenyl)ethyl]-5-[(1E)-2-(2,6-dihydroxyphenyl)ethenyl]-1,3-benzenediol (2) and 4-(6,8-dimethoxyl-2-naphthalenyl)1,3-benzenediol (3). Their structures were elucidated on the basis of spectral analysis, and their possible formation mechanisms were discussed. $\mathbf{1}$ and $\mathbf{2}$ were new linear stilbene dimers synthesized for the first time. Pharmacological tests showed $\mathbf{1}$, 2 and 3 to exhibit potent anti-oxidation activity with $\mathrm{IC}_{50}$ values of $6.29 \times 10^{-9}, 4.19 \times 10^{-6}$, and $2.96 \times 10^{-5} \mathrm{~mol} \cdot \mathrm{L}^{-1}$, respectively, and 2 was shown to have potent anti-inflammatory activity.

Keywords gnetol dimer; oxidative coupling reaction; bioactivity
\end{abstract}

文献报道二苯乙烯及其低聚茋类化合物表现出多 种多样的生物活性, 如抗炎、抗氧化、抗菌、抗 HIV 及 抗肿瘤等 ${ }^{[1]}$. 尤其是异丹叶大黄素和白㢣芦醇对白三烯 的生成抑制和受体拮抗显示了很强的生物活性 ${ }^{[2]}$. 近年 来, 随着对植物活性成分的深入研究, 越来越多的天然 低聚茋类化合物被分离出来, 包括白藜芦醇低聚体、异 丹叶大黄素低聚体以及氧化白㢣芦醇低聚体等. 同时, 对它们的生物活性也进行了广泛的研究, 并发现了一系 列活性较好的低聚茋类化合物 ${ }^{[3]}$. 买麻藤醇是从买麻藤 属植物中分离得到的一个二苯乙烯单体化合物, 药理实 验表明, 它具有较强的抗炎和抗氧化活性. 然而, 迄今 为止, 除了 gnetuhainin $\mathrm{K}($ 买麻藤醇和异丹叶大黄素形
成的二聚体 $)^{[4]}$, 在天然界还没有发现别的买麻藤醇低 聚茋类化合物. 在前期的研究中, 本研究组 ${ }^{[\sim 9]}$ 曾先后 报道了异丹叶大黄素低聚体和白㢣芦醇低聚体的合成 和制备. 为了获得结构类型多样的低聚茋类化合物进行 生物活性篮选，以发现活性更强的化合物作为进一步深 入研究开发的候选药物, 同时深入研究买麻藤醇聚合反 应的规律和特点, 本研究设计对从闭苞买麻藤中获得的 买麻藤醇单体的聚合反应进行研究. 以 $\mathrm{FeCl}_{3} \cdot 6 \mathrm{H}_{2} \mathrm{O}$ 为 氧化剂, 买麻藤醇在甲醇溶液中进行氧化偶联反应获得 两个新的买麻藤醇二聚体：4-[1-(2,6-二羟基苯基)-2(3,5-二羟基苯基)乙基]-2-[(1E)-2-(3,5-二羟基苯基)乙烯 基]-1,3-苯二醇(1)和 2-[1-(2,6-二羟基苯基)-2-(3,5-二羟

*E-mail: linmao@imm.ac.cn

Received October 29, 2012; revised November 17, 2012; published online November 26, 2012. 
基苯基)乙基]-5-[(1E)-2-(2,6-二羟基苯基)乙烯基]-1,3-苯 二醇(2)(图 1). 买麻藤醇单体在甲醇溶液中进行酸催化 聚合, 反应混合物经分离获得一个新的苯基菜衍生物 4-(6,8-二甲氧基-2-荎基)-1,3-苯二醇(3) (图 1). 应用波谱 分析的方法(包括 UV, IR, MS, ${ }^{1} \mathrm{H}$ NMR, ${ }^{13} \mathrm{C}$ NMR 以及 2D NMR 等)确定了它们的结构, 并分别讨论了它们可 能的形成机理. 其中, 化合物 $\mathbf{1}$ 和 $\mathbf{2}$ 为两个新的天然产 物类似物, 为首次人工合成的二苯乙烯链状二聚体. 化 合物 3 为新型的买麻藤醇二聚体失去两个苯环生成的苯 基萗衍生物. 药理活性测试结果表明, 化合物 $\mathbf{1} \sim 3$ 均显 示有较强的抗氧化活性, 化合物 2 还显示有较强的抗炎 活性.

\section{1 结果与讨论}

以闭苞买麻藤 (Gnetum cleistostachyum) 中分离获得 的天然买麻藤醇为原料, $\mathrm{FeCl}_{3} \cdot 6 \mathrm{H}_{2} \mathrm{O}$ 为氧化剂在甲醇中 室温摚拌反应 $10 \mathrm{~d}$, 反应混合物经硅胶柱层析分离, 获 得两个新的天然产物类似物: 化合物 1 和化合物 2 . 另 外, 将买麻藤醇在 $5 \%$ 硫酸甲醇溶液中加热回流 $48 \mathrm{~h}$, 经硅胶柱层析分离获得一个新结构的菜衍生物 3 (图 1), 它们的结构分别鉴定如下.

化合物 1, 灰色无定形粉末. 其质谱 FAB MS $m / z$ (\%)中可观察到准分子离子峰 $489\left([\mathrm{M}+\mathrm{H}]^{+}, 17\right)$, 分子 离子峰 $488\left(\mathrm{M}^{+}, 10\right)$, 结合 ${ }^{1} \mathrm{H}$ NMR 谱和 ${ }^{13} \mathrm{C}$ NMR 谱数 据(表 1), 推断化合物 $\mathbf{1}$ 的分子式为 $\mathrm{C}_{28} \mathrm{H}_{24} \mathrm{O}_{8}$, 提示化合 物 $\mathbf{1}$ 可能为一个买麻藤醇的二聚体. $\mathbf{1}$ 的紫外光谱在 $\lambda_{\text {max }}$ $(\log \varepsilon): 226$ (4.59)和 312 (4.42) nm 处有吸收, 显示分子 结构中有较强的共轭系统存在; IR 光谱显示有羟基 $\left(v_{\text {max }}\right.$ $\left.3340 \mathrm{~cm}^{-1}\right)$, 苯环和双键的吸收 $\left(v_{\text {max }} 1693,1597,1464\right.$ $\mathrm{cm}^{-1}$ ). 化合物 1 的 ${ }^{1} \mathrm{H}$ NMR 谱显示有一个 $7 \mathrm{a}$ 位取代的 二氢买麻藤醇结构单元: $\delta 6.35(\mathrm{~d}, J=7.8 \mathrm{~Hz}, 2 \mathrm{H}, \mathrm{H}-3 \mathrm{a}$, $5 \mathrm{a})$ 和 $6.78(\mathrm{t}, J=7.8 \mathrm{~Hz}, 1 \mathrm{H}, \mathrm{H}-4 \mathrm{a})$ 的一套属于环 $\mathrm{A}_{1}$ 的 $\mathrm{AB}_{2}$ 系统; $\delta 6.25$ (brs, 2H, H-10a, 14a) 和 6.08 (brs, 1H, $\mathrm{H}-12 \mathrm{a})$ 的一套属于环 $\mathrm{A}_{2}$ 的 $\mathrm{AB}_{2}$ 系统以及在 $\delta 4.86(\mathrm{t}, J=$ $7.2 \mathrm{~Hz}, 1 \mathrm{H}, \mathrm{H}-7 \mathrm{a}), 3.67$ (dd, $J=7.2,13.8 \mathrm{~Hz}, 1 \mathrm{H}, \mathrm{H}-8 \mathrm{a} \alpha$ ) 和 $3.43(\mathrm{dd}, J=7.2,13.8 \mathrm{~Hz}, 1 \mathrm{H}, \mathrm{H}-8 \mathrm{a} \beta)$ 的三个相偶合的 脂肪氢信号. 此外，化合物 $\mathbf{1}$ 的 ${ }^{1} \mathrm{H}$ NMR 谱中还观察到 另外一个 $3 \mathrm{~b}$ 位取代的买麻藤醇的氢信号: $\delta 7.46(\mathrm{~d}, J=$ $7.5 \mathrm{~Hz}, 1 \mathrm{H}, \mathrm{H}-4 \mathrm{~b})$ 和 $6.42(\mathrm{~d}, J=7.5 \mathrm{~Hz}, 1 \mathrm{H}, \mathrm{H}-5 \mathrm{~b})$ 的一套 属于环 $\mathrm{B}_{1}$ 的 $\mathrm{AB}$ 系统氢信号; $\delta 6.48$ (brs, $2 \mathrm{H}, \mathrm{H}-10 \mathrm{~b}, 14 \mathrm{~b}$ ) 和 6.21 (brs, $1 \mathrm{H}, \mathrm{H}-12 \mathrm{~b}$ ) 的一套属于环 $\mathrm{B}_{2}$ 的 $\mathrm{AB}_{2}$ 系统氢 信号; 同时, 在 $\delta 7.38(\mathrm{~d}, J=16.8 \mathrm{~Hz}, 1 \mathrm{H})$ 和 $7.50(\mathrm{~d}, J=$ $16.8 \mathrm{~Hz}, 1 \mathrm{H}$ )处显示有两个相偶合的烯氢质子信号. 化 合物 1 的 ${ }^{13} \mathrm{C}$ NMR 谱显示有 20 个碳信号代表 28 个碳 (13 个季碳, 其中 6 个被氧化; 14 个叔碳以及一个仲碳).
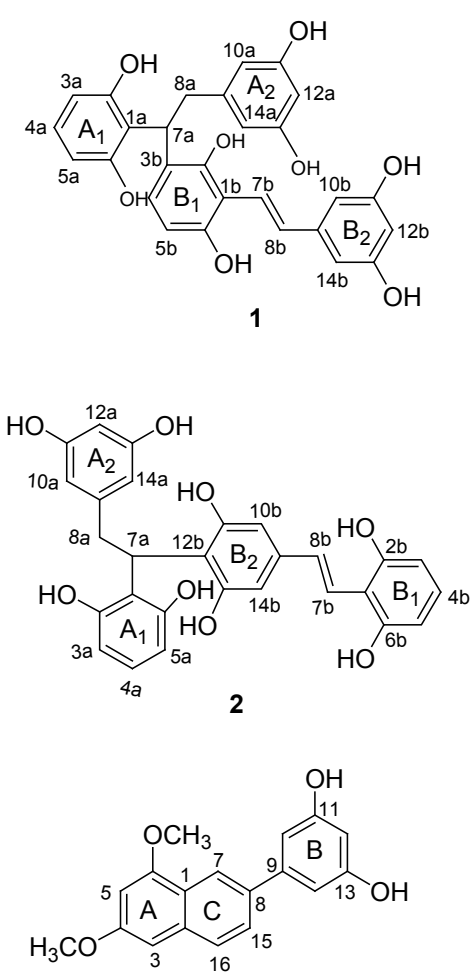

3

图 1 化合物 1,2 和 3 的结构

Figure 1 Structures of 1, 2 and 3

综合以上光谱数据推断, 化合物 $\mathbf{1}$ 为一个 $7 \mathrm{a}$ 位取代的二 氢买麻藤醇和一个 $3 \mathrm{~b}$ 位取代的买麻藤醇相连接的买麻 藤醇二聚体, 它与天然产物 artogomezianol(从植物 Artocarpus gomezianus 分离获得)具有相同的分子骨 架 ${ }^{[10]}$. 为了进一步确证两个买麻藤醇结构单元的连接 位置, 进行了 HMBC 实验(图 2). 其中, H-7a 与 C-2(6)a, C-9a，C-2b，C-4b 相关，H-8a 和 C-1a，C-10(14)a 相关, $\mathrm{H}-5 \mathrm{~b}$ 与 $\mathrm{C}-1 \mathrm{~b}$ 和 $\mathrm{C}-3 \mathrm{~b}$ 相关进一步证实两个买麻藤醇结 构单元通过 7a-C 与 $3 \mathrm{~b}-\mathrm{C}$ 相连. 因此, 化合物 $\mathbf{1}$ 的结构 确定为 4-[1-(2,6-二羟基苯基)-2-(3,5-二羟基苯基)乙 基]-2-[(1E)-2-(3,5-二羟基苯基)乙烯基]-1,3-苯二醇，命 名为双买麻藤醇 $\mathrm{A}$.

化合物 2, 灰色无定形粉末. 其质谱 FAB MS m/z (\%)给出准分子离子峰 $489\left([\mathrm{M}+\mathrm{H}]^{+}, 25\right)$ 以及分子离子 峰 $488\left(\mathrm{M}^{+}, 17\right)$, 结合 ${ }^{1} \mathrm{H}$ NMR 谱和 ${ }^{13} \mathrm{C}$ NMR 谱数据(表 1), 推断其分子式为 $\mathrm{C}_{28} \mathrm{H}_{24} \mathrm{O}_{8}$, 提示化合物 2 可能为一 个买麻藤醇的二聚体. $\mathrm{UV}$ 光谱显示在 $\lambda_{\max }(\log \varepsilon): 226$ (3.48), 313 (4.33)和 324 (4.33) nm 有吸收, 显示分子结 构中存在强的共轭系统. IR 光谱显示有羟基 $\left(v_{\max } 3320\right.$ $\left.\mathrm{cm}^{-1}\right)$, 苯环和双键的吸收 $\left(v_{\text {max }} 1697,1614,1464 \mathrm{~cm}^{-1}\right)$. 与化合物 $\mathbf{1}$ 相似，化合物 $\mathbf{2}$ 的 ${ }^{1} \mathrm{H}$ NMR 谱也观察到一个 $7 \mathrm{a}$ 位取代的二氢买麻藤醇结构单元: $\delta 6.43(\mathrm{~d}, J=8.1$ 
表 1 化合物 1 和 $\mathbf{2}$ 的 ${ }^{1} \mathrm{H} \mathrm{NMR}$ 和 ${ }^{13} \mathrm{C}$ NMR 谱数据 ${ }^{a}$

Table $1{ }^{1} \mathrm{H}$ NMR and ${ }^{13} \mathrm{C}$ NMR sectral data of $\mathbf{1}$ and 2

\begin{tabular}{|c|c|c|c|c|c|}
\hline \multirow{2}{*}{ Position } & \multicolumn{2}{|l|}{1} & \multirow{2}{*}{ Position } & \multicolumn{2}{|l|}{2} \\
\hline & $\delta_{\mathrm{H}}$ & $\delta_{\mathrm{C}}$ & & $\delta_{\mathrm{H}}$ & $\delta_{\mathrm{C}}$ \\
\hline $1 \mathrm{a}$ & & 117.2 & $1 \mathrm{a}$ & & 117.3 \\
\hline $2 \mathrm{a}$ & & 154.6 & $2 \mathrm{a}$ & & 156.1 \\
\hline $3 a$ & $6.35(\mathrm{~d}, J=7.8 \mathrm{~Hz})$ & 107.9 & $3 a$ & $6.43(\mathrm{~d}, J=8.1 \mathrm{~Hz})$ & 109.3 \\
\hline $4 a$ & $6.78(\mathrm{t}, J=7.8 \mathrm{~Hz})$ & 127.7 & $4 a$ & $6.82 \sim 6.91(\mathrm{~m})$ & 128.5 \\
\hline $5 \mathrm{a}$ & $6.35(\mathrm{~d}, J=7.8 \mathrm{~Hz})$ & 107.9 & $5 \mathrm{a}$ & $6.43(\mathrm{~d}, J=8.1 \mathrm{~Hz})$ & 109.3 \\
\hline $6 a$ & & 154.6 & $6 a$ & & 156.1 \\
\hline $7 \mathrm{a}$ & $4.86(\mathrm{t}, J=7.2 \mathrm{~Hz})$ & 35.8 & $7 \mathrm{a}$ & $5.12(\mathrm{t}, J=7.5 \mathrm{~Hz})$ & 34.6 \\
\hline $8 \mathrm{a} \alpha$ & $3.67(\mathrm{dd}, J=7.2,13.8 \mathrm{~Hz})$ & & $8 \mathrm{a} \alpha$ & $3.59(\mathrm{dd}, J=7.5,13.8 \mathrm{~Hz})$ & \\
\hline $8 \mathrm{a} \beta$ & $3.43(\mathrm{dd}, J=7.2,13.8 \mathrm{~Hz})$ & 37.6 & $8 \mathrm{a} \beta$ & $3.50(\mathrm{dd}, J=7.5,13.8 \mathrm{~Hz})$ & 37.3 \\
\hline $9 \mathrm{a}$ & & 144.6 & $9 \mathrm{a}$ & & 144.5 \\
\hline $10 \mathrm{a}$ & 6.25 (brs) & 107.9 & $10 \mathrm{a}$ & $6.23(\mathrm{~d}, J=1.5 \mathrm{~Hz})$ & 107.6 \\
\hline $11 \mathrm{a}$ & & 158.6 & $11 \mathrm{a}$ & & 158.7 \\
\hline $12 \mathrm{a}$ & 6.08 (brs) & 100.7 & $12 \mathrm{a}$ & 6.09 (brs) & 100.9 \\
\hline $13 a$ & & 158.6 & $13 \mathrm{a}$ & & 158.7 \\
\hline $14 \mathrm{a}$ & 6.25 (brs) & 107.9 & $14 \mathrm{a}$ & $6.23(\mathrm{~d}, J=1.5 \mathrm{~Hz})$ & 107.6 \\
\hline $1 b$ & & 121.3 & $1 b$ & & 112.5 \\
\hline $2 b$ & & 155.1 & $2 b$ & & 157.3 \\
\hline $3 b$ & & 112.3 & $3 b$ & $6.43(\mathrm{~d}, J=8.1 \mathrm{~Hz})$ & 106.9 \\
\hline $4 b$ & $7.46(\mathrm{~d}, J=7.5 \mathrm{~Hz})$ & 121.8 & $4 \mathrm{~b}$ & $6.82 \sim 6.91(\mathrm{~m})$ & 128.2 \\
\hline $5 b$ & $6.42(\mathrm{~d}, J=7.5 \mathrm{~Hz})$ & 107.1 & $5 b$ & $6.43(\mathrm{~d}, J=8.1 \mathrm{~Hz})$ & 106.9 \\
\hline $6 b$ & & 155.1 & $6 b$ & & 157.3 \\
\hline $7 b$ & $7.38(\mathrm{~d}, J=16.8 \mathrm{~Hz})$ & 128.8 & $7 b$ & $7.38(\mathrm{~d}, J=16.5 \mathrm{~Hz})$ & 121.1 \\
\hline $8 b$ & $7.50(\mathrm{~d}, J=16.8 \mathrm{~Hz})$ & 131.4 & $8 b$ & $7.46(\mathrm{~d}, J=16.5 \mathrm{~Hz})$ & 130.9 \\
\hline $9 b$ & & 142.4 & $9 b$ & & 139.9 \\
\hline $10 \mathrm{~b}$ & 6.48 (brs) & 105.1 & $10 \mathrm{~b}$ & 6.61 (brs) & 107.6 \\
\hline $11 \mathrm{~b}$ & & 159.1 & $11 \mathrm{~b}$ & & 156.1 \\
\hline $12 b$ & 6.21 (brs) & 101.8 & $12 \mathrm{~b}$ & & 115.9 \\
\hline $13 b$ & & 159.1 & $13 b$ & & 156.1 \\
\hline $14 \mathrm{~b}$ & 6.48 (brs) & 105.1 & $14 \mathrm{~b}$ & $6.61(\mathrm{brs})$ & 107.6 \\
\hline
\end{tabular}

${ }^{a}$ Measured in $\mathrm{CD}_{3} \mathrm{COCD}_{3}$ at $500 \mathrm{MHz}$ for ${ }^{1} \mathrm{H}$ NMR and $125 \mathrm{MHz}$ for ${ }^{13} \mathrm{C}$ NMR.
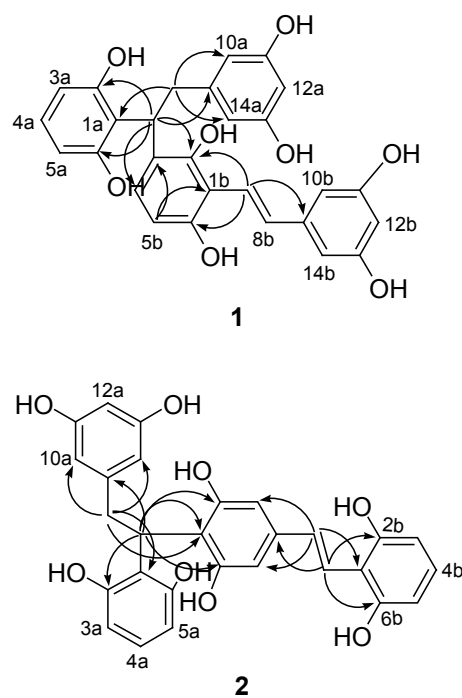

图 2 化合物 1 和 2 结构中关键的 HMBC 相关信号

Figure 2 Significant HMBC interactions of $\mathbf{1}$ and $\mathbf{2}$

$\mathrm{Hz}, 2 \mathrm{H}, \mathrm{H}-3 \mathrm{a}, 5 \mathrm{a})$ 和 $6.82 \sim 6.91(\mathrm{~m}, 1 \mathrm{H}, \mathrm{H}-4 \mathrm{a})$ 为一套属于 环 $\mathrm{A}_{1}$ 的 $\mathrm{AB}_{2}$ 系统氢信号, $\delta 6.23(\mathrm{~d}, J=1.5 \mathrm{~Hz}, 2 \mathrm{H}, \mathrm{H}-10 \mathrm{a}$,
$14 \mathrm{a})$ 和 6.09 (brs, $1 \mathrm{H}, \mathrm{H}-12 \mathrm{a})$ 为另一套属于环 $\mathrm{A}_{2}$ 的 $\mathrm{AB}_{2}$ 系统氢信号, $\delta 5.12(\mathrm{t}, J=7.5 \mathrm{~Hz}, 1 \mathrm{H}, \mathrm{H}-7 \mathrm{a}), 3.59(\mathrm{dd}, J=$ 7.5, $13.8 \mathrm{~Hz}, 1 \mathrm{H}, \mathrm{H}-8 \mathrm{a} \alpha)$ 和 $3.50(\mathrm{dd}, J=7.5,13.8 \mathrm{~Hz}, 1 \mathrm{H}$, $\mathrm{H}-8 \mathrm{a} \beta)$ 为 3 个相偶合的脂肪氢信号. 化合物 2 的 ${ }^{1} \mathrm{H}$ NMR 谱还显示存在另一套 $12 \mathrm{~b}$ 位取代的买麻藤醇结构单元: $\delta$ $6.43(J=8.1 \mathrm{~Hz}, 2 \mathrm{H}, \mathrm{H}-3 \mathrm{~b}, 5 \mathrm{~b})$ 和 $6.82 \sim 6.91(\mathrm{~m}, 1 \mathrm{H}$, $\mathrm{H}-4 \mathrm{~b}$ )为一套属于环 $\mathrm{B}_{1}$ 的 $\mathrm{AB}_{2}$ 系统氢信号, $\delta 6.61$ (brs, $2 \mathrm{H}, \mathrm{H}-10 \mathrm{~b}, 14 \mathrm{~b})$ 为一个单峰代表环 $\mathrm{B}_{2}$ 的两个氢信号, 以 及 $\delta 7.38(\mathrm{~d}, J=16.5 \mathrm{~Hz}, 1 \mathrm{H}, \mathrm{H}-7 \mathrm{~b})$ 和 $7.46(\mathrm{~d}, J=16.5 \mathrm{~Hz}$, $1 \mathrm{H}, \mathrm{H}-8 \mathrm{~b})$ 的两个相偶合的双键质子信号. 同时，化合物 2 的 ${ }^{13} \mathrm{C}$ NMR 谱显示有 20 个信号代表 28 个碳(13 个季 碳, 其中 8 个被氧化, 14 个叔碳信号以及一个仲碳信号). 这些光谱数据提示化合物 2 应该是一个买麻藤醇二聚 体，它与天然产物 andalasin $\mathrm{A}$ (从植物 Artocarpus gomezianus 分离获得)具有相似的分子骨架 ${ }^{[10]}$. 在 $\mathrm{HMBC}$ 谱中(图 2), H-7a 与 C-2(6)a, C-9a, C-12b, C-11(13)b 相关, $\mathrm{H}-8 \mathrm{a}$ 与 C-1a, C-10(14)a 和 C-12b 相关, 进一步证实两个 买麻藤醇结构单元通过 $\mathrm{C}-7 \mathrm{a}$ 与 $\mathrm{C}-12 \mathrm{~b}$ 相连. 因此, 化 
合物 2 的结构确定为 2-[1-(2,6-二羟基苯基)-2-(3,5-二羟 基苯基)乙基]-5-[(1E)-2-(2,6-二羟基苯基)乙烯基]-1,3-苯 二醇, 命名为双买麻藤醇 $\mathrm{B}$.

化合物 3, 浅黑色无定形粉末. 其质谱 FAB MS $m / z$ $296\left(\mathrm{M}^{+}\right)$, 结合 ${ }^{1} \mathrm{H}$ NMR 谱和 ${ }^{13} \mathrm{C}$ NMR 谱(表 2)数据分 析推测分子式为 $\mathrm{C}_{18} \mathrm{H}_{16} \mathrm{O}_{4}$, 提示分子中有 11 个不饱和 度. 化合物 3 的 UV 光谱显示在 $\lambda_{\text {max }} 224,257$ 和 $304 \mathrm{~nm}$ 处有吸收, 提示分子中有较强的共轭系统存在. 其 IR 光 谱显示有羟基 $\left(v_{\text {max }} 3370 \mathrm{~cm}^{-1}\right)$ 和苯环 $\left(v_{\max } 1601,1500\right.$, $1454 \mathrm{~cm}^{-1}$ )的吸收. 化合物 3 的 ${ }^{1} \mathrm{H}$ NMR 谱显示存在一 套属于环 $\mathrm{C}$ 的 $\mathrm{ABX}$ 系统氢信号 $[\delta 7.79(\mathrm{~d}, J=8.7 \mathrm{~Hz}$, $1 \mathrm{H}), 7.67(\mathrm{dd}, J=8.7,2.1 \mathrm{~Hz}, 1 \mathrm{H})$ 和 $8.28(\mathrm{~d}, J=2.1 \mathrm{~Hz}$, $1 \mathrm{H})]$; 一套属于环 $\mathrm{B}$ 的 $\mathrm{AB}_{2}$ 系统氢信号 $[\delta 6.36(\mathrm{t}, J=2.1$ $\mathrm{Hz}, 1 \mathrm{H})$ 和 $6.73(\mathrm{~d}, J=2.1 \mathrm{~Hz}, 2 \mathrm{H})]$; 两个属于环 $\mathrm{A}$ 的相 偶合的芳香氢信号 $[\delta 6.87(\mathrm{~d}, J=2.1 \mathrm{~Hz}, 1 \mathrm{H})$ 和 $6.58(\mathrm{~d}$, $J=2.1 \mathrm{~Hz}, 1 \mathrm{H})]$; 以及两个属于甲氧基的氢信号 $[\delta 4.01$ $(\mathrm{s}, 3 \mathrm{H})$ 和 $3.90(\mathrm{~s}, 3 \mathrm{H})]$. 化合物 3 的 ${ }^{13} \mathrm{C} \mathrm{NMR}$ 谱在低场 区 $\delta 98 \sim 160$ 显示有 16 个芳香碳信号, 在 $\delta 56.1$ 和 56.6 显示有两个甲氧基的碳信号. 综合以上数据推测 3 为一 个 3,5-二羟基苯基取代菜的衍生物(图 1). 为了确定两个 甲氧基的取代位置, 进行了 NOE 实验(图 3). 当分别照 射 H-3 和 H-5 时, 两个甲氧基的氢信号分别产生了增益, 揭示两个甲氧基分别取代在 C-4 和 C-6 位上. HMBC 谱 (图 3)中, H-10(14)和 C-8, C-12, C-11(13)相关, H-3 和 C-1, C-4, C-16 相关, H-5 和 C-1, C-4, C-6 相关进一步证 实了以上推断. 因此, 化合物 3 的结构确定为 4-(6,8-二 甲氧基-2-萗基)-1,3-苯二醇, 3 为一个新的苯基荟衍生物.

化合物 1 和 2 为两个新的买麻藤醇二聚体, 其可能 的形成机理推断如下: 在氧化偶联反应过程中, 买麻藤 醇被 $\mathrm{FeCl}_{3} \cdot 6 \mathrm{H}_{2} \mathrm{O}$ 氧化形成酚羟基自由基中间体 $\mathbf{R}_{2} \cdot, \mathbf{R}_{3} \cdot$, $\mathbf{R}_{\mathbf{1 1}}$ 和 $\mathbf{R}_{\mathbf{1 2}} \bullet$. 其中, 间位的酚羟基自由基 $\mathbf{R}_{\mathbf{1 1}}$ ・不能够与 双键以及另一个苯环产生共轭作用, 因而稳定性较低, 反应活性不高; 邻位酚羟基自由基 $\mathbf{R}_{2}$ - 虽然能与双键和 另一个苯环产生较好的共轭作用, 有较高的稳定性, 但 是由于两个邻位羟基引起的较大空间位阻作用, 大大降 低了买麻藤醇双键的反应活性(在同样反应条件下, 与 白僽芦醇和异丹叶大黄素相比); 另一方面, 两个邻位 羟基的存在使得苯基自由基 $\mathbf{R}_{\mathbf{3}}$ •和 $\mathbf{R}_{\mathbf{1 2}}$ • 更加稳定, 因此, 反应中产生的氢自由基优先结合到活性较高、位阻较小 的 C-8a 位(邻位羟基产生的位阻使其难以接近 C-7a 位), 从而产生 $\mathbf{R}_{7} \cdot$ 自由基(图 4).

基于以上推断, 化合物 $\mathbf{1}$ 和 $\mathbf{2}$ 的形成过程可解释如 下(图 5): 自由基 $\mathbf{R}_{\mathbf{3}} \bullet$ 和 $\mathbf{R}_{\mathbf{7}}$ 偶联产生不稳定的醌式中间 体 $\mathbf{a}, \mathbf{a}$ 通过氢转移生成了结构稳定的化合物 1. 同理, 自由基 $\mathbf{R}_{\mathbf{7}} \bullet$ 和 $\mathbf{R}_{\mathbf{1 2}} \cdot$ 偶联产生不稳定的醌式中间体 $\mathbf{b}, \mathbf{b}$ 异
表 2 化合物 3 的 ${ }^{1} \mathrm{H} \mathrm{NMR}$ 和 ${ }^{13} \mathrm{C}$ NMR 数据 ${ }^{a}$

Table $2{ }^{1} \mathrm{H}$ NMR and ${ }^{13} \mathrm{C}$ NMR spectral data of 3

\begin{tabular}{|c|c|c|}
\hline Position & $\delta_{\mathrm{H}}$ & $\delta_{\mathrm{C}}$ \\
\hline 1 & & 122.5 \\
\hline 2 & & 135.5 \\
\hline 3 & $6.87(\mathrm{~d}, J=2.1 \mathrm{~Hz})$ & 98.6 \\
\hline 4 & & 159.5 \\
\hline 5 & $6.58(\mathrm{~d}, J=2.1 \mathrm{~Hz})$ & 98.8 \\
\hline 6 & & 157.5 \\
\hline 7 & $8.28(\mathrm{~d}, J=2.1 \mathrm{~Hz})$ & 120.1 \\
\hline 8 & & 136.3 \\
\hline 9 & & 144.2 \\
\hline 10 & $6.73(\mathrm{~d}, J=2.1 \mathrm{~Hz})$ & 106.3 \\
\hline 11 & & 159.9 \\
\hline 12 & $6.36(\mathrm{t}, J=2.1 \mathrm{~Hz})$ & 102.3 \\
\hline 13 & & 159.9 \\
\hline 14 & $6.73(\mathrm{~d}, J=2.1 \mathrm{~Hz})$ & 106.3 \\
\hline 15 & $7.67(\mathrm{dd}, J=8.7,2.1 \mathrm{~Hz})$ & 126.9 \\
\hline 16 & $7.79(\mathrm{~d}, J=8.7 \mathrm{~Hz})$ & 127.9 \\
\hline $\mathrm{OCH}_{3}$ & $4.01(\mathrm{~s})$ & 56.6 \\
\hline $\mathrm{OCH}_{3}$ & $3.90(\mathrm{~s})$ & 56.1 \\
\hline
\end{tabular}
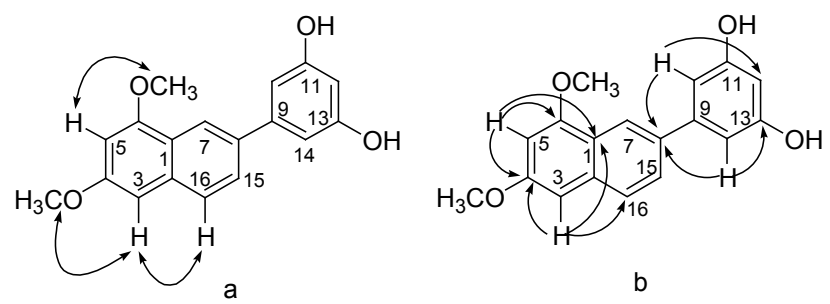

图 3 化合物 3 结构中重要的 NOE (a) 和 HMBC (b) 相关信号 Figure 3 Important NOE (a) and HMBC (b) correlations of 3

构化为结构稳定的化合物 2(图 5). 异丹叶大黄素和白藜 芦醇由于存在对位羟基的共轭作用，用金属氧化剂进行 氧化偶联时容易产生不同结构类型的自由基中间体，因 而偶联产物复杂, 产物类型多 ${ }^{[5,6]}$. 而买麻藤醇缺少对位 差基的共轭作用，同时，邻位羟基产生较大的空间位阻. 因此, 在同样的条件下, 反应需要进行很长时间, 而且 产生的自由基中间体类型较少，从而导致产物数量和类 型均较少。

化合物 3 的形成过程可解释为两个买麻藤醇单体 通过 Diels-Alder 加成产生的二聚体再失去两个苯环形 成的苯基荟衍生物 ${ }^{[7]}$. 两分子买麻藤醇通过头尾相连的 方式加成产生不稳定的苯并环已烯中间体 $\mathbf{c}, \mathbf{c}$ 连续失去 两个苯环生成了稳定的具有较长共轭体系的化合物 $\mathbf{e}$. 同时，在甲醇溶液中，两个羟基与甲醇中的甲基进行交 换生成了化合物 3(图 6). 很明显, 两个邻位酚羟基的存 在活化了芳环, 使得相应的中间体 $\mathbf{c}$ 和 $\mathbf{d}$ 稳定性降低, 很容易失去两个苯环生成更稳定的化合物 e. 因为在本 反应中, $\mathbf{c}$ 和 $\mathbf{d}$ 均没有分离获得. 


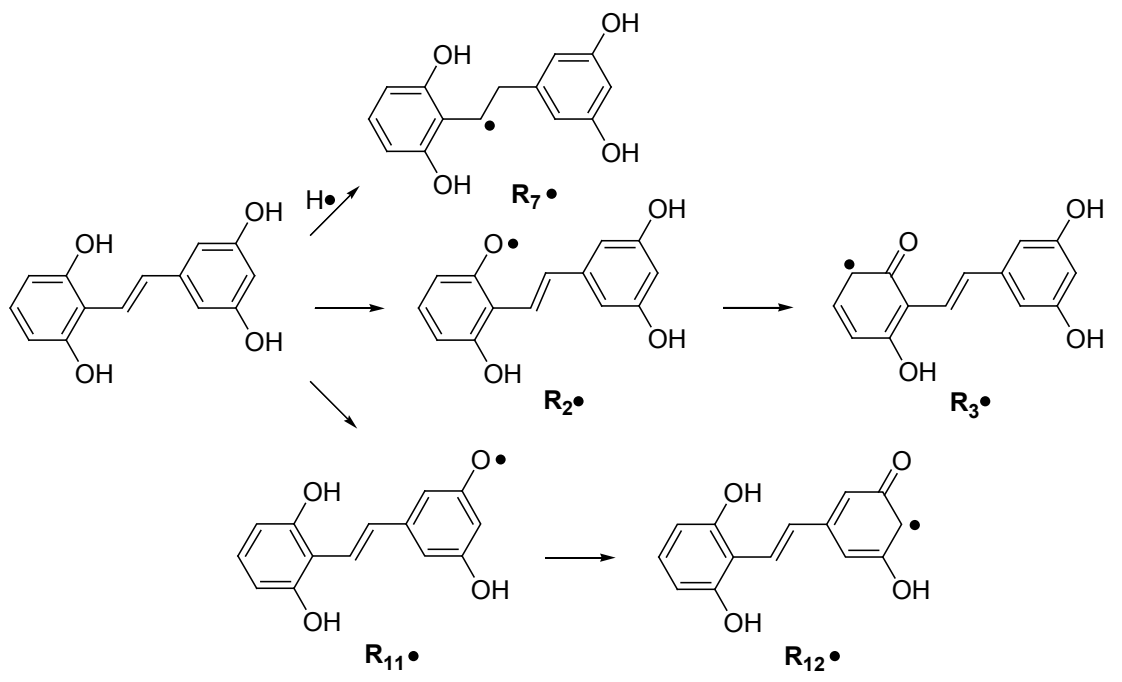

图 4 买麻藤醇氧化偶联反应中可能的自由基中间体

Figure 4 Possible free radicals in the oxidative coupling reaction of gnetol

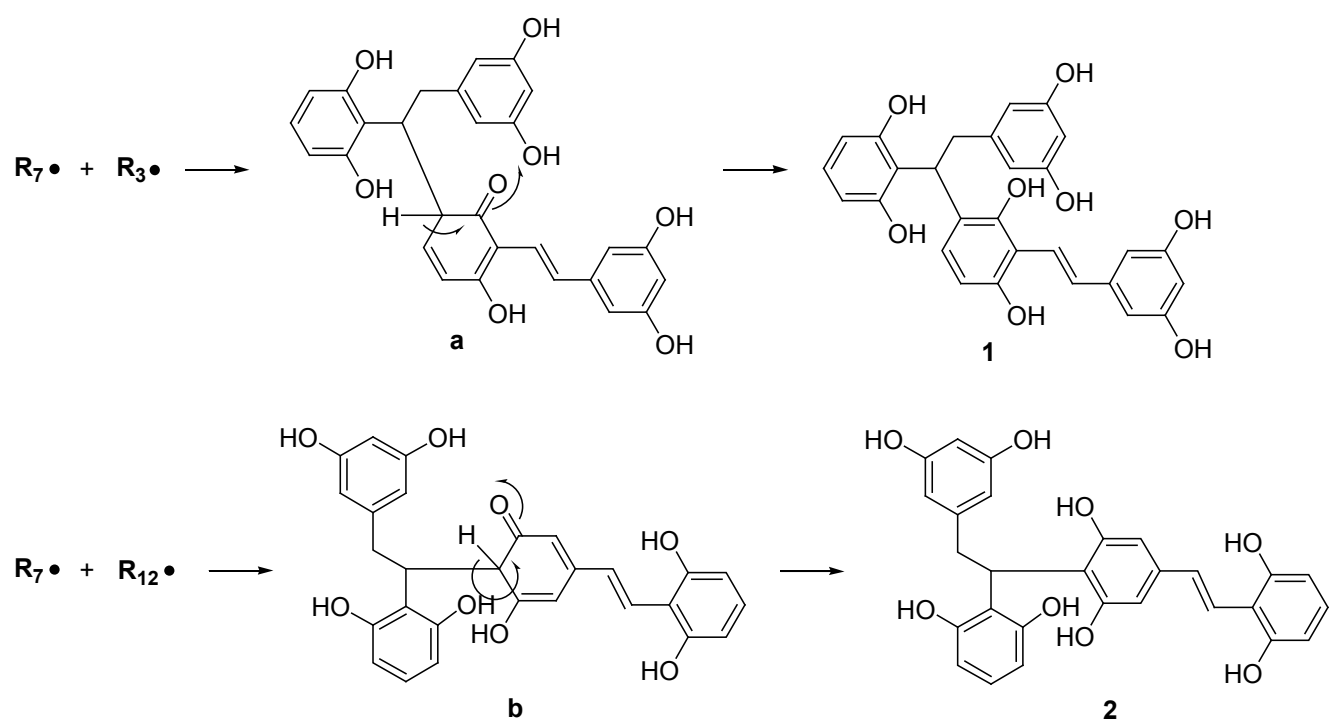

图 5 化合物 $\mathbf{1}$ 和 $\mathbf{2}$ 形成过程中可能的中间体

Figure 5 Postulated intermediates in the formation of compounds 1 and 2

对化合物 1 3 进行了初步的药理活性测试(表 3), 结果表明, 在 $10^{-5} \mathrm{~mol} / \mathrm{L}$ 条件下, 化合物 1 3 对小鼠腹 腔巨噬细胞引起的 $\mathrm{TNF} \alpha$ 生成抑制率分别为 $17.43 \%$ ，40.26\%和 7.34\% (地塞米松为阳性对照，其对 $\mathrm{TNF} \alpha$ 抑制的 $\mathrm{IC}_{50}$ 值为 $1 \times 10^{-6} \mathrm{~mol} \cdot \mathrm{L}^{-1}$ ). 同时, 化合物

表 3 化合物 1,2 和 3 对 TNF $\alpha$ 和 MDA 的抑制活性 ${ }^{a}$

Table 3 TNF $\alpha$ and MDA inhibitory activities of $\mathbf{1}, \mathbf{2}$, and $\mathbf{3}$

\begin{tabular}{cccc}
\hline Compound & $\mathbf{1}$ & $\mathbf{2}$ & $\mathbf{3}$ \\
\hline $\mathrm{TNF} \alpha$ inhibitory rate ${ }^{b} / \%$ & -17.43 & 40.26 & 7.34
\end{tabular}

MDA inhibitory activity

$\left[\mathrm{IC}_{50}\left(\mathrm{~mol} \cdot \mathrm{L}^{-1}\right)\right]$

$6.29 \times 10^{-9} 4.19 \times 10^{-6} 2.96 \times 10^{-5}$

${ }^{a}$ Dexamethasone was used as a positive control for $\mathrm{TNF} \alpha$ with $\mathrm{IC}_{50}$ of $1 \times$ $10^{-6} \mathrm{~mol} \cdot \mathrm{L}^{-1}$, and Vit E was used for MDA with $\mathrm{IC}_{50}$ of $1 \times 10^{-6} \mathrm{~mol} \cdot \mathrm{L}^{-1}$. ${ }^{b}$ Concentration: $1 \times 10^{-5} \mathrm{~mol} \cdot \mathrm{L}^{-1}$.
$1 \sim 3$ 对丙二醛(MDA)生成抑制的 $\mathrm{IC}_{50}$ 值分别为 $6.29 \times$ $10^{-9}, 4.19 \times 10^{-6}$ 以及 $2.96 \times 10^{-5} \mathrm{~mol} \cdot \mathrm{L}^{-1}$ (Vit E 为阳性 对照, 其对 $\mathrm{MDA}$ 抑制的 $\mathrm{IC}_{50}$ 值为 $1 \times 10^{-6} \mathrm{~mol} \cdot \mathrm{L}^{-1}$ ). 以 上结果显示, 化合物 2 具有很强的抗炎活性, 化合物 1 3 具有较强的抗氧化活性.

\section{2 结论}

以 $\mathrm{FeCl}_{3} \cdot 6 \mathrm{H}_{2} \mathrm{O}$ 为氧化剂, 天然买麻藤醇单体为原 料, 在甲醇溶液中进行氧化偶联反应, 获得了两个新的 买麻藤醇二聚体衍生物 $\mathbf{1}$ 和 2 . 它们均为两个通过 $\mathrm{C}-\mathrm{C}$ 键相连的新的买麻藤醇二聚体. 然而, 在反应过程中没 有获得通过 $\mathrm{C}-\mathrm{O}$ 键相连的化合物, 表明买麻藤醇的氧 化偶联反应与异丹叶大黄素和白藜芦醇是不同的. 后者 
<smiles>Oc1cc(O)c(-c2c(-c3c(O)cccc3O)cc3cc(O)cc(O)c3c2-c2c(O)cccc2O)c(O)c1</smiles>

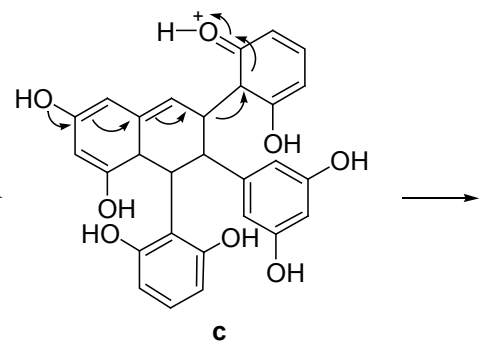

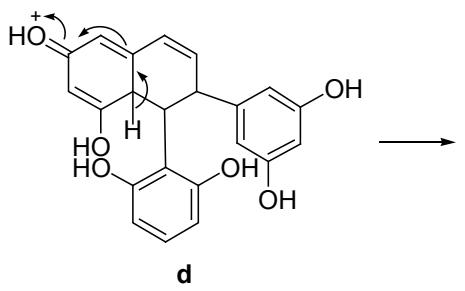

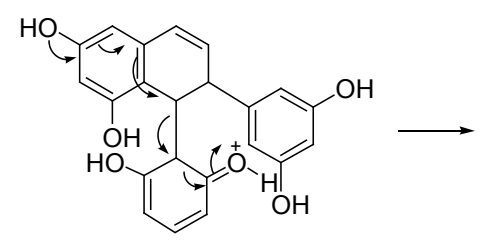<smiles>CC1=C2C=CC(=O)C=C2C=CC1c1cc(O)cc(O)c1</smiles><smiles>Oc1cc(O)cc(-c2ccc3cc(O)cc(O)c3c2)c1</smiles><smiles>COc1cc(OC)c2cc(-c3cc(O)cc(O)c3)ccc2c1</smiles>

图 6 化合物 3 形成过程中可能的中间体

Figure 6 Plausible intermediates in the formation of compound 3

在同样的条件下, 可获得 $\mathrm{C}-\mathrm{C}$ 键和 $\mathrm{C}-\mathrm{O}$ 键相连的结 构复杂的化合物. 在买麻藤醇的氧化偶联反应中, 位阻 起了关键作用. 有关这个反应更多的情况, 还需要进行 更深入的研究, 以便获得更多的有关反应活性和反应机 理的证据. 另外, 买麻藤醇在硫酸催化条件下进行二聚 反应, 获得了一个新型的脱除两个苯基的苯基荟衍生物 3, 说明在酸催化反应中, 买麻藤醇与白著芦醇和异丹 叶大黄素有着相似的反应性质, 位阻并不是反应中的关 键性因素. 化合物 1 3 均为天然产物类似物, 它们表现 出了较好的抗炎活性和抗氧化活性. 金属和酸催化的二 苯乙烯氧化偶联反应在制备活性二苯乙烯化合物方面 是一个有意义的值得深入研究的方法.

\section{3 实验部分}

\section{1 仪器与试剂}

熔点用 Kofler HB 熔点仪测定; 旋光用 Perkin-Elmer digital polarimeter 旋光仪测定; IR 光谱用 Perkin-Elmer 683 红外光谱仪测定, $\mathrm{KBr}$ 压片; UV 光谱用 Shimadzu UV-300 光谱仪测定; NMR 谱用 AM 500 型核磁共振仪 测定, TMS 为内标; HPLC 为 waters 411 型, 配有 waters 2487 检测器以及 waters 515 洜; EI-MS 和 HR-FAB-MS 均在 QB-200 质谱仪上测定.

\section{2 实验方法}

3.2.1 以 $\mathrm{FeCl}_{3} \cdot 6 \mathrm{H}_{2} \mathrm{O}$ 为氧化剂的买麻藤醇氧化偶联 反应

摚拌下, $3.88 \mathrm{~g} \mathrm{FeCl}_{3} \cdot 6 \mathrm{H}_{2} \mathrm{O}(14.34 \mathrm{mmol})$ 的水溶液
逐滴滴加到买麻藤醇 $(2.70 \mathrm{~g}, 11.07 \mathrm{mmol})$ 的甲醇溶液中, 室温搅拌反应 $10 \mathrm{~d}$. 反应混合物减压浓缩, 浓缩物悬浮 于水中, 以 EtOAc 萃取 3 次, 合并萃取液, $\mathrm{Na}_{2} \mathrm{SO}_{4}$ 干燥, 减压浓缩. 所得浓缩物硅胶柱层析, 环己烷丙酮 $(V$ : $V=5: 1 \sim 4: 1$ )洗脱得 $\mathrm{G} 1 \sim \mathrm{G} 3$ 三部分, $\mathrm{G} 1$ 部分为未反 应原料买麻藤醇 $(1.40 \mathrm{~g}) . \mathrm{G} 2$ 部分进行硅胶柱层析, $\mathrm{CHCl}_{3}-\mathrm{MeOH}(V: V=25: 1)$ 洗脱得化合物 $2(64 \mathrm{mg}$, 产率 5\%). G3 部分经硅胶柱层析, $\mathrm{CHCl}_{3}-\mathrm{MeOH}(V$ : $V=10: 1)$ 洗脱得化合物 $\mathbf{1}(115.7 \mathrm{mg}$, 产率 $9 \%)$.

4-[1-(2,6-二羟基苯基)-2-(3,5-二羟基苯基)乙基]-2[(1E)-2-(3,5-二羟基苯基)乙烯基]-1,3-苯二醇(1): 灰色无 定形粉末, 产率 9\%. m.p. 212 214 ${ }^{\circ} \mathrm{C}$; UV-Vis $(\mathrm{MeOH})$ $\lambda_{\text {max }}(\log \varepsilon): 226$ (4.59), $312(4.42) \mathrm{nm} ;{ }^{1} \mathrm{H}$ NMR 和 ${ }^{13} \mathrm{C}$ NMR 谱数据见表 1; IR (KBr) $v_{\text {max }}$ : 3340, 1693，1597, 1464, 1340, 1290, 1238, 1147, 1063, 993, 833, 791, 683 $\mathrm{cm}^{-1}$; FAB MS $m / z(\%): 489\left([\mathrm{M}+\mathrm{H}]^{+}, 17\right), 488\left(\mathrm{M}^{+}\right.$, 10), 365 (8), 282 (11), 245 (8), 185 (64), 93 (100), 75 (24), 57 (53), 45 (33). HR-FAB-MS calcd for $\mathrm{C}_{28} \mathrm{H}_{24} \mathrm{O}_{8}$ 488.1471, found 488.1492 .

2-[1-(2,6-二羟基苯基)-2-(3,5-二羟基苯基)乙基]5-[(1E)-2-(2,6-二羟基苯基)乙烯基]-1,3-苯二醇(2): 灰色 无定形粉末，产率 5\%. m.p. $201 \sim 203{ }^{\circ} \mathrm{C}$; UV-Vis $(\mathrm{MeOH}) \lambda_{\max }(\log \varepsilon): 226$ (3.48), 313 (4.33), 324 (4.33) $\mathrm{nm} ;{ }^{1} \mathrm{H}$ NMR 和 ${ }^{13} \mathrm{C}$ NMR 数据见表 1; IR (KBr) $v_{\text {max }}$ : 3320, 1697, 1614, 1464, 1338, 1236, 1161, 1055, 995, 829, $777,717 \mathrm{~cm}^{-1}$; FAB MS $m / z(\%): 489\left([\mathrm{M}+\mathrm{H}]^{+}, 25\right), 488$ 
$\left(\mathrm{M}^{+}, 17\right), 391$ (18), 365 (27), 282 (12), 245 (18), 185 (54), 154 (47), 137 (57), 107 (27), 93 (100), 57 (31). HR-FABMS calcd for $\mathrm{C}_{28} \mathrm{H}_{24} \mathrm{O}_{8} 488.1471$, found 488.1489.

\section{2 .2 酸催化的买麻藤醇二聚反应}

买麻藤醇 $\left(259 \mathrm{mg}\right.$ ) 溶于 $40 \mathrm{~mL} 5 \% \mathrm{H}_{2} \mathrm{SO}_{4}$ 甲醇溶液 中, 搅拌下加热回流 $48 \mathrm{~h}$. 停止反应, 反应混合物中加 入 $150 \mathrm{~mL}$ 水, EtOAc 萃取 $(50 \mathrm{~mL} \times 3)$, 有机层合并, $\mathrm{Na}_{2} \mathrm{SO}_{4}$ 干燥, 减压浓缩. 所得浸膏状物硅胶柱层析, $\mathrm{CHCl}_{3}-\mathrm{MeOH}(V: V=500: 1)$ 洗脱得 4-(6,8-二甲氧基2-萘基)-1,3-苯二醇(3) (60 mg, 38.2\%). 浅黑色无定形粉 末, m.p. 162 164 ${ }^{\circ} \mathrm{C}$; UV-Vis $(\mathrm{MeOH}) \lambda_{\max }: 224,257$, $304 \mathrm{~nm} ;{ }^{1} \mathrm{H}$ NMR 和 ${ }^{13} \mathrm{C}$ NMR 谱数据见表 2; IR (KBr) $v_{\max }: 3370,2935,2827,1699,1628,1601,1500,1454$, 1402, 1342, 1286, 1207, 1159, 1111, 1045, 997, 926, 833, 696, $671 \mathrm{~cm}^{-1}$; FAB MS m/z (\%): $296\left(\mathrm{M}^{+}, 100\right), 185$ (10), 132 (17), 93 (24), 69 (14). HR-FAB-MS calcd for $\mathrm{C}_{18} \mathrm{H}_{16} \mathrm{O}_{4} 296.1049$, found 296.1088.

3.2 .3 抗炎和抗氧化活性测定

抗炎和抗氧化活性测定均依据文献[11, 12]提供的
方法进行.

致谢 中国医学科学院药物研究所程桂芳教授代测抗 炎活性, 刘耕陶教授代测抗氧化活性, 在此一并致谢.

\section{References}

[1] Shimizu, K.; Kondo, R.; Sakai, K. Planta Med. 2000, 66, 11.

[2] Xu, G.; Zhang, L. Y.; Chen, L. F.; Hu, C. Q. Acta Pharm. Sin. 1994, 29,818 .

[3] Li, N.; Li, X. M.; Huang, K. S.; Lin, M. Acta Pharm. Sin. 2001, 36, 944.

[4] Wang, Y. H.; Huang, K. S.; Lin, M. J. Asian Nat. Prod. Res. 2001, 3,169 .

[5] Zhou, L. X.; Lin, M. Acta Pharm. Sin. 2000, 35, 669.

[6] Yao, C. S.; Zhou, L. X.; Lin, M. Chem. Pharm. Bull. 2004, 52, 238.

[7] Li, X. M.; Huang, K. S.; Lin, M.; Zhou, L. X. Tetrahedron 2003, $59,4405$.

[8] Huang, K. S.; Lin, M.; Wang, Y. H. Chin. Chem. Lett. 1999, 10, 817.

[9] Zhou, L. X.; Lin, M. Chin. Chem. Lett. 2000, 11, 515.

[10] Likhitwitayawuid, K.; Sritularak, B. J. Nat. Prod. 2001, 64, 1457.

[11] Li, J.; Cheng, G. F.; Zhu, X. Y. Acta Pharm. Sin. 2000, 35, 335.

[12] Dai, S. J.; Ma, Z. B.; Wu, Y.; Chen, R. Y.; Yu, D. Q. Phytochemistry 2004, 65, 3135 . 